ISSN 2590-9770

The Art of Discrete and Applied Mathematics 1 (2018) \#P2.04

https://doi.org/10.26493/2590-9770.1239.e1f

(Also available at http://adam-journal.eu)

\title{
Reaction graphs of double Fano planes
}

\author{
Mariusz Meszka \\ AGH University of Science and Technology, Kraków, Poland \\ Alexander Rosa \\ McMaster University, Hamilton, ON, Canada \\ Dedicated to Mario Gionfriddo on the occasion of his 70th birthday.
}

Received 3 January 2018, accepted 16 February 2018, published online 6 August 2018

\begin{abstract}
We consider various reaction graphs on the set of distinct double Fano planes.
\end{abstract}

Keywords: Double Fano plane, reaction graph, strongly regular graph.

Math. Subj. Class.: 05B07, 05C62

The concept of a reaction graph, which has its origin in chemistry, has been explored in several papers, for example, [5, 7, 8, 9, 10, 11].

The reaction graph(s) of the Fano plane (i.e. projective plane of order 2, Steiner triple system of order 7 , or $\operatorname{BIBD}(7,3,1))$ are considered in detail in $[8,9]$, with some additional comments provided in [7]. The vertices of such reaction graph are the 30 distinct Fano planes (on a fixed 7-element set). The reaction graph is of degree 14,8, and 7, respectively, according to how adjacency is defined: namely, whenever two vertices (Fano planes) have one, zero, or three triples in common, respectively. The graph of degree 14 is actually isomorphic to $2 K_{15}$, that is, two disjoint complete graphs $K_{15}$ as components. Each of these corresponds to a maximal set of MAD STS(7)s (mutually almost disjoint STSs, cf. [6]).

It is well known that the simple $\operatorname{BIBD}(7,3,2)$ (i.e. with no repeated blocks) is unique up to an isomorphism and consists of two disjoint Fano planes. It contains 14 blocks (triples) and its automorphism group is of order 42 . The blocks (triples) of one such design can be represented as $\{0,1,3\},\{0,2,3\} \bmod 7$. We shall call any simple $\operatorname{BIBD}(7,3,2)$ a double Fano plane. Thus there are $\frac{7 !}{42}=120$ distinct double Fano planes on any 7 -element set. A double Fano plane will be denoted $(a, b)$ provided $a$ and $b$ are the two disjoint Fano planes that constitute it.

Let $(a, b),(c, d)$ be two distinct double Fano planes. Due to the structure of the reaction graphs of the single Fano plane, whenever $|\{a, b, c, d\}|=4$, two of the 4 intersections

E-mail addresses: meszka@agh.edu.pl (Mariusz Meszka),rosa@mcmaster.ca (Alexander Rosa) 
between $a$ and $c, a$ and $d, b$ and $c$, and $b$ and $d$ must contain exactly one triple. Without loss of generality we may assume that the intersection between $a$ and $c$, and also between $b$ and $d$ both contain one triple. The remaining two intersections, namely between $a$ and $d$, and between $b$ and $c$, may

(i) both contain zero triples, or

(ii) both contain three triples, or

(iii) one contains zero and the other contains three triples.

The edges of our reaction graph on $K_{120}$ can now be one of four kinds: either $|\{a, b, c, d\}|=3$, or it is one of the three types above (see Figure 1).

We shall use the following "colourful" terminology.

A green edge joins two double Fano planes $(a, b),(c, d)$ when $|\{a, b, c, d\}|=3$, that is, one of $a, b$ equals one of $c, d$. A yellow, blue or red edge, respectively, joins two double Fano planes $(a, b),(c, d)$ when $|\{a, b, c, d\}|=4$ and case (i), (ii) or (iii), respectively, occurs.

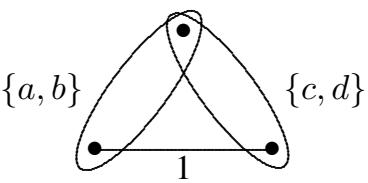

green edge

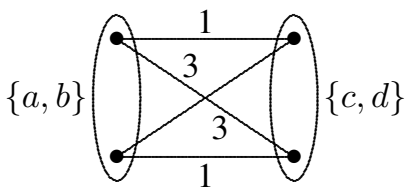

blue edge

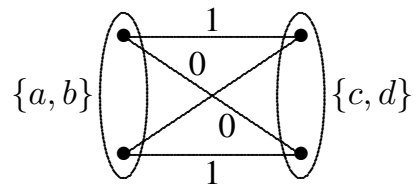

yellow edge

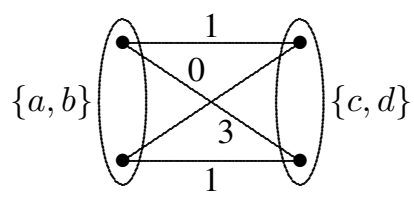

red edge

Figure 1: Pairs of double Fano planes.

According to the aforementioned intersections, one may define the following four reaction graphs:

I. The green graph (the subgraph of $K_{120}$ induced by the green edges).

This graph is regular of degree 14 , and is quasi-strongly regular of grade 3 (cf. [4]) with parameters $(120,14,6,(0,1,2))$.

II. The yellow graph (the subgraph of $K_{120}$ induced by the yellow edges).

This graph is regular of degree 21 , and is quasi-strongly regular of grade 2 (cf. [4]) with parameters $(120,21,0,(3,6))$.

III. The blue graph (the subgraph of $K_{120}$ induced by the blue edges).

This graph is regular of degree 28 and is quasi-strongly regular of grade 3 with parameters $(120,28,6,(4,6,12))$. 
IV. The red graph (the subgraph of $K_{120}$ induced by the red edges).

This graph is regular of degree 56 . and is strongly regular with parameters $(120,56$, $28,24)$. A strongly regular graph with these parameters and automorphism group of order 348364800 is known to exist (cf. [3]; see also [12, 13]).

The four coloured graphs together form a 4-class association scheme. The intersection numbers for this scheme can be found at http://home.agh.edu.pl/ meszka/ reaction_graphs.html.

Next we want to investigate the structure of so-called neighbourhood graphs.

For a vertex $\{a, b\}$ of the reaction graph, a vertex $\{c, d\}$ joined to it by a green edge is called a green neighbour, and similarly for yellow, blue or red edges we have yellow, blue, or red neighbours.

Given a vertex of the reaction graph, the green neighbourhood graph is the complete graph $K_{14}$ on its green neighbours. Its edges are coloured green, yellow or red - there are no blue edges. The green edges induce graph consisting of two disjoint $K_{7}$ 's, the yellow edges induce the Heawood graph (cf. [1]), and the red edges induce the bipartite complement of the Heawood graph. It is well-known that the automorphism group of the Heawood graph is $\operatorname{PGL}(2,7)$ of order 336 . The coloured edges form a 3-class association scheme.

The yellow neighbourhood graph of a vertex is the complete graph $K_{21}$ on its yellow neighbours. Its edges are 3-coloured: green, blue and red; there are no yellow edges. The graph induced by the green edges is regular of degree 4 and is distance-transitive with intersection array $[4,2,2 ; 1,1,2]$, with automorphism group $\operatorname{PGL}(2,7)$ of order 336 . The graphs induced by the blue and red edges, respectively, both have degree 8 , and the same automorphism group as the graph induced by green edges. In this case too the coloured edges form a 3-class association scheme.

The blue neighbourhood graph of a vertex is the complete graph $K_{28}$ on its blue neighbours. Its edges are 4-coloured. The graph induced by green and blue edges, respectively, is regular of degree 6 , while the graph induced by the yellow edges is cubic, and is actually isomorphic to the Coxeter graph (cf. [2]). The automorphism group of each of these three graphs is again $\operatorname{PGL}(2,7)$. The graph induced by the red edges is the so-called 8triangular graph; it is regular of degree 12 , and is distance-transitive with intersection array $[12,5 ; 1,4]$. Its automorphism group is $S_{8}$ of order 40320 . The coloured edges form a 4-class association scheme.

Finally, the red neighbourhood graph of a vertex is the complete graph $K_{56}$ on its red neighbours. Its edges are 4-coloured. The graph induced by green edges is of degree 6 , and its automorphism group has order 225792 . Those induced by the yellow, blue and red edges, respectively, are of degree 9,12 , and 28 , respectively, where the first two of these have automorphism group $\operatorname{PGL}(2,7)$ of order 336 , while the last one has large automorphism group of order 2903040 . In this case, the coloured edges do not form an association scheme.

Let us remark that the graph induced by the union of the green and blue edges is of degree 42 , and turns out to be a quasi-strongly regular graph of grade 2 (cf. [4]) with parameters $(120,42,18,(6,15))$. Of course, the graph induced by the union of green, yellow and blue edges is complementary to the red graph, and so is strongly regular with parameters $(120,63,30,36)$. 


\section{References}

[1] A. E. Brouwer, A. M. Cohen and A. Neumaier, Distance-Regular Graphs, volume 18 of Ergebnisse der Mathematik und ihrer Grenzgebiete, Springer-Verlag, Berlin, 1989, doi: 10.1007/978-3-642-74341-2.

[2] H. S. M. Coxeter, My graph, Proc. London Math. Soc. 46 (1983), 117-136, doi:10.1112/plms/ s3-46.1.117.

[3] D. Crnković, S. Rukavina and A. Švob, New strongly regular graphs from orthogonal groups $\mathrm{O}^{+}(6,2)$ and $\mathrm{O}^{-}(6,2)$, Discrete Math. 341 (2018), 2723-2728, doi:10.1016/j.disc.2018.06. 029.

[4] F. Goldberg, On quasi-strongly regular graphs, Linear Multilinear Algebra 54 (2006), 437-451, doi:10.1080/03081080600867210.

[5] M. H. Klin, S. S. Tratch and N. S. Zefirov, Group-theoretical approach to the investigation of reaction graphs for highly degenerate rearrangements of chemical compounds: I. Criterion of the connectivity of a graph, J. Math. Chem. 7 (1991), 135-151, doi:10.1007/bf01200820.

[6] C. C. Lindner and A. Rosa, Construction of large sets of almost disjoint Steiner triple systems, Canad. J. Math. 27 (1975), 256-260, doi:10.4153/cjm-1975-031-3.

[7] C. C. Lindner and A. Rosa, Reaction graphs of the $K_{4}-e$ design of order 6, Bull. Inst. Combin. Appl. 47 (2006), 43-47.

[8] E. K. Lloyd, The reaction graph of the Fano plane, in: T.-H. Ku (ed.), Combinatorics and Graph Theory '95, Volume 1, World Scientific Publishing Co., River Edge, NJ, pp. 260-274, 1995, proceedings of the Summer School and International Conference on Combinatorics (SSICC '95) held in Hefei, May 25 - June 5, 1995.

[9] E. K. Lloyd, Some graphs associated with the seven point plane, Amer. J. Math. Management Sci. 20 (2000), 85-101, doi:10.1080/01966324.2000.10737502.

[10] E. K. Lloyd and G. A. Jones, Reaction graphs, Acta Appl. Math. 52 (1998), 121-147, doi: 10.1023/a:1005954907636.

[11] Z. Masárová, Reaction graphs for some complete graph decompositions, to appear in J. Combin. Math. Combin. Comput.

[12] R. Mathon and A. P. Street, Overlarge sets and partial geometries, J. Geom. 60 (1997), 85-104, doi:10.1007/bf01252220.

[13] R. Mathon and A. P. Street, Partitions of sets of designs on seven, eight and nine points, $J$. Statist. Plann. Inference 58 (1997), 135-150, doi:10.1016/s0378-3758(96)00066-3. 